\title{
Annotatio et Erratum
}

\section{A Physical Interpretation of the Cusp Conditions for Molecular Wave Functions}

Theoret. chim. Acta (Berl.) 8, 54 (1967)

\author{
W. A. BINGEL
}

Lehrstuhl für Theoretische Chemie der Universität Göttingen

Received September 29, 1967

The third term in Eq. (21), page 60, should be

$$
\left(-\frac{1}{4} \Delta_{0}-\sum_{\alpha} \frac{2 Z_{\alpha}}{r_{0 \alpha}}\right) \quad \text { instead of } \quad\left(-\frac{1}{2} \Delta_{0}-\sum_{\alpha} \frac{Z_{\alpha}}{r_{0 \alpha}}\right) \text {. }
$$

The following remark may be added.

The expansion (22) can be simplified for 2 -electron systems. If $S=0$, the spatial wave function must be symmetric with respect to a permutation of electrons 1 and 2 . This means, that the second term in (22) must vanish $(c \equiv 0)$. If $S=1$, the spatial wave function must be antisymmetric, with the result that the first term in (22) vanishes identically $\left[\Psi\left(\boldsymbol{r}_{0}, \boldsymbol{r}_{\mathbf{0}}, \ldots\right) \equiv 0\right]$.

PACK and Byers-Brown (Ref. [3] of my paper) state, that this alternative still obtains for $n$-electron systems with any $n>2$. This statement needs some clarification. Only in the case of maximal spin $S=n / 2$, where $\Psi$ must be antisymmetric in all electrons, we can still say that the first term in Eq. (22) must always vanish identically. For $S<n / 2$, there are $f_{S}^{n}$ different spatial wave functions $\Psi_{i}$ for the same energy, which span their reducible representation $\mathfrak{D}_{S}^{n}$ of the permutation group. They can indeed be so chosen, that the matrix representation of the special permutation $P_{12}$ is diagonal and has diagonal elements equal to +1 or -1 . For those $\Psi_{i}$ which belong to rows with -1 the first term and for those which belong to rows with +1 the second term in their expansion (22) must vanish. However, there is no longer any alternative, since both cases occur in the expression

$$
\Phi_{S, M_{S}}=\sum_{i=1}^{f_{S}^{n}} \Psi_{i} \Theta_{S, M_{S}, i}
$$

for the complete wave function of a single state including spin. Moreover, any other choice of the degenerate basis, while leaving (2) invariant, would lead to a situation, where the $\Psi_{i}$ are neither symmetric nor antisymmetric with respect to interchange of electrons 1 and 2.

Prof. Dr. W. A. Bingert

Lehrstuhl für Theoretische Chemie

der Universität Göttingen

3400 Göttingen

Bürgerstr. 50a 